\title{
DAVID Y GOLIAT: LA INFERIORIDAD NUMÉRICA DE LOS CASTELLANOS EN EL POEMA DE FERNÁN GONZÁLES
}

\author{
Alí Viquez Jiménez
}

\begin{abstract}
RESUMEN
Este artículo estudia el proceso de reescritura del personaje bíblico de David dentro del Poema de Fernán Gonzáles. Basado en la examinación contextual del pasaje bíblico, el estudio pretende analizar la evidencia de inferioridad numérica en las batallas peleadas por los castellanos liderados por Fernán Gonzáles. A través de un análisis de estas victorias castellanas y sus causas, se llega a ciertas conclusiones del impacto ideológico de la comparación entre David y Fernán Gonzáles.
\end{abstract}

\begin{abstract}
This article studies the process of rewriting of the Biblical character David within the Poema de Fernán Gonzáles. Based on contextual examination of the Biblical passage, the study intends to analyze the evidence of numerical inferiority in the battles fought by the Castillians led by Fernán Gonzáles. Through an analysis of these Castillian victories and their causes, the study then arrives at certain conclusions of the ideological impact of the comparison between David and Fernán Gonzáles.
\end{abstract}

\section{Introducción}

Este trabajo versa sobre el Poema de Fernán Gonzáles (en adelante, Poema), redactado en Castilla durante el siglo XIII. Se sabe, por las referencias que en las Crónicas se han encontrado, que existió un cantar juglaresco dedicado al fundador de Castilla, y muy anterior al Poema que estudiamos. Tal cantar se ha perdido. Es importante señalar desde el principio la diferencia existente entre un cantar juglaresco de carácter popular y una composición propia del mester de clerecía. El Arlantino, nombre convencionalmente dado al autor del Poema, no escribió su texto al calor de los acontecimientos narrados, en un tiempo en el que Castilla se hallaba todavía como el incipiente proyecto de una nación. Al contrario, el Arlantino vive una época en la que la superioridad castellana se ha consolidado relativamente en la península y Fernán Gonzáles no vive fresco en la memoria de las personas.

Esto no quiere decir, sin embargo, que la fortaleza de Castilla tuviera entonces un carácter autosuficiente. Es tradicional, en primer lugar, que las naciones dominantes reescriban 
su historia en procura de contar con los instrumentos ideológicos que justifiquen la dominación, siempre cuestionable. Dicho de otro modo, si del cantar perdido (esto, claro, en ausencia de un texto que lo verifique, es parcialmente hipotético) se podía esperar una orientación ideológica pro dominación de Castilla, al calor del nacimiento de la región, del Poema también podemos esperar algo similar, con la diferencia de que se trata ahora de justificar una dominación dada en el presente. En segundo lugar, aunque Castilla ya domine, no lo hace sin oposición ni sin que nuevos proyectos expansionistas se encuentren en agenda. Es probable que el Arlantino compusiera este poema durante el periodo de auge de la reconquista castellana en el que reinó Fernando III. Esta es, al menos, la tesis que defiende Victorio (1988:27). Partiendo del hecho de que Fernando es izado como modelo de gobernante, su eficacia será to-
tal si le imita un monarca real. Un monarca que dé gran impulso a la Reconquista (hasta el pun-
to de ser sobrenominado Santo), que reine en esos mismos años, que se llame igual que el pro-
tagonista del Poema, y que también gobierne a los castellanos. Ese no es otro que Fernando III,
muerto en 1252 .

También O’Callagham trae a colación el proyecto militar que caracterizó el reinado de Fernando III: "Fernando won extraordinary renown because of his conquest of most Andalusia and Murcia” (14).

Tomando, pues, como marco general de referencia una orientación ideológica pro Castilla de consolidación y continuación de un proyecto expansionista, este trabajo quiere detenerse en lo que puede ser una particular estrategia ideológica dentro de dicho marco general. Queremos examinar una posible reelaboración de un pasaje bíblico, la historia de David y Goliat (1 y 2, Samuel), en el Poema, dada específicamente a través de una homologación entre David y el héroe Fernán Gonzáles, representante del pueblo castellano. El énfasis lo pondremos en el tópico de la inferioridad numérica de los castellanos liderados por Fernán Gonzáles, en las batallas narradas en el texto.

Tres citas textuales justifican inicialmente nuestro interés. La primera se halla en la copla 107 (todas las citas del Poema en este trabajo provienen de la edición de Juan Victorio):

Señor, tu que libreste a Davit del leon, mateste al Filisteo, un sobervio varon, quiteste a los jodios del rey de Babilon, saca nos e libra nos de tal cruel presion.

Esta cita quizá no ofrece una prueba demasiado sólida de la importancia de la historia de David en el texto, ya que se encuentra dentro de una oración pronunciada por los castellanos antes de la aparición en el Poema del héroe, y al final dicha petición no surte efecto, pues los castellanos no logran rechazar a los moros. Además, se hallan allí enumeradas una serie de historias bíblicas, y esta parte no resalta.

La segunda cita es la copla 270:

Por nos detener en otras ledanias,

fue Almaçor vençido con sus cavallerias:

allí fue demostrado el poder del Mexias,

el conde fue David y Almançor Gollias. 
Aquí sí nos parece evidente la importancia de la comparación. (Aunque el nombre de David sea una reconstrucción del editor, en este caso tal reconstrucción es indiscutiblemente acertada.) El narrador focalizador externo homologa a David y a Fernán Gonzáles, lo que demuestra que la comparación tiene bases en la voz más autorizada del relato, es decir, la del narrador.

Finalmente, tenemos la copla 354, en la que el propio Fernán Gonzáles (sin duda, la segunda voz más autorizada en el texto, y la primera dentro del mundo narrado) se focaliza a sí mismo y a los suyos en la figura de David:

\footnotetext{
Non cuentan d'Alexandre las noches nin los dias, cuentan sus buenos fechos e sus cavallerias; cuentan del rey Davit que mato a Golias, de Judas Macabeo, fijo de Matatias.
}

Aquí es importante señalar que la autofocalización se hace en relación con la fama futura: los castellanos han de entrar en combate para que se los recuerde como se recuerda a David, entre otros.

Nos detendremos primeramente en los estudios que pueden servir de antecedente a este trabajo, como estado de la cuestión. Luego dedicaremos un apartado a la Biblia en la Edad Media, y en particular a la lectura que de la historia de David se hizo durante ese periodo. En tercer lugar, intentaremos un examen detallado de los pasajes en los que se narran batallas, para confirmar que la inferioridad numérica de los castellanos es un dato establecido por el texto mismo. En cuarto lugar, examinaremos los procedimientos mediante los cuales los castellanos obtienen la victoria: aquí estudiaremos la arenga del conde y su estrategia retórica, así como la intervención divina en pro de los castellanos y la oposición demoniaca. En quinto lugar, esbozaremos las conclusiones.

\section{Antecedentes}

Como antecedentes de este trabajo, hemos encontrado solamente el artículo de Cotrait (1973: 359-82), cuya primera parte se dedica más bien a otro texto (David perseguido y Alivio de Lastimados), pero cuya segunda parte promete dedicarse a un problema cercano al que aquí nos hemos planteado. Dice Cortait:

Une question se pose, en effet, capitale pour quiconque s'interroge sur les origines et les sources de la légende gonzalienne et sur ses succesives manifestations littérraires: c'est de savoir si l'episode biblique de David et Michol a pu exercer quelque influence sur celui de Fernán Gonzáles et doña Sancha, voire présider à sa gestation (373).

Ahora bien, Cotrait se centra en un episodio particular de la historia bíblica de David, que no es el enfrentamiento con Goliat, sobre el cual nosotros hemos puesto el énfasis. No obstante, sus argumentos son importantes a la hora de justificar nuestro trabajo. Así, después de señalar algunas divergencias entre el texto bíblico y la versión del poema, dice Cotrait:

Mais elles n'affectent en rien la structure fondamentale commune aux deux épisodes. C'est, en effet, le meme schéma qui les sous-tend: $\left({ }^{\circ}\right)$ On voit d'ailleurs assez bien par quel mécanisme 
a pu s'opérer le transfert, de l'histoire de David à celle de Fernán Gonzáles, du narrème de la libération par la fille de l'ennemi. L'identité de leurs rapports avec leur entourage respectif, la similitude de leur comportement, rapprochent les deux héros, au point que dans le Poema le comte de Castille nous apparaisse comme une réplique castillane de David. Philistins ou Musulmans, tous deux défendent pareillement l'intégrité territoriale de leur nation contre l'étranger, et dans le deux cas cet adversaire est aussi l'ennemi de la foi. Tous deux sont en butte à l'hostilité des rois qui, par plusieurs fois, tentent de les réduire à l'impuissance. Tous deux enfin triomphent de l'adversité avec l'aide de Dieu. L'auteur du Poema ne s'y pas trompé, qui prend David pour parangon supreme des vertus du comte castillan (377).

\section{La biblia y David en la Edad Media}

Según Riché, es indudable la utilización de las Sagradas Escrituras como argumento de autoridad durante la Edad Media: "A chaque époque du Moyen Age, tous les dirigeants, clercs et laïcs, ont utlisé les arguments scripturaires pour donner autorité à leurs principes de gouvernement et à leurs revendications" (385).

Aunque Riché no se refiere en particular al periodo de la historia peninsular que nos interesa, sí establece ideas de carácter general que justifican la orientación de este trabajo. Así dice: 'Depuis l'établissement de l'Empire chrétien, il n'est pas rare de trouver sous la plume des évêques du Conseil des Empereurs des parallèles entre ceux qui sont lieutenants de Dieu sur la terre et les figures de l'Ancien Testament" (385). Y aunque al principio de la Edad Media la Biblia compartía su influencia con otras fuentes, según Riché "à partir du VIIème siècle, la Bible et la Bible seule devient le livre de référence par excellence pour cuex qui refléchissent à la condition royale" (386).

La verificación de esta tesis la ofrece Riché precisamente en relación con el caso de la Península Ibérica, aunque en una época muy anterior a la que nos ocupa:

Nous le constatons dans l'Espagne wisigothique dans laquelle le clergé joue un role politique prédominant. Les conciles nationaux de Tolède sont des assemblées autant religieuses que politiques. Les èvêques wisigoths et particulièrement Isidore de Seville imaginent la royauté wisigothique à l'exemple de celle que l'Ancien Testamente présente (386).

Así pues, encontramos justificado el pensar que David pueda leerse como uno de los modelos -no el único, por supuesto- a partir de los cuales se ha construido el personaje de Fernán Gonzáles y, metonímicamente, la imagen del pueblo castellano, en el Poema.

Por otra parte, y más específicamente en relación con la figura de David, Frontain y Wojcik confirman la importancia que ha tenido esta en Occidente, y particularmente durante la Edad Media:

For the pre-Renaissance world -from the time of the earliest religious interpretations through the European Middle Ages- David's significance was a type, one of the richest and most fully exploited (...) For Christian audiences David is a type of Christ, the assumption being that Jesus of Nazareth was the Messiah who, it was prophetized, would sit on David's trhone and reestablish his lost kingdom (...) Like David, Christ was a shepherd -but of souls, not actual sheep. David's triumphs against adversity, especially in his defeat of the giant Goliath, were seen to foreshadow Christ's victories against Satan (2-3). 
Nos interesa particularmente resaltar la tesis de Charles A. Huttar, que puede sernos muy útil a la hora de elaborar nuestras propias conclusiones. Este investigador pone el énfasis en David como modelo de arrepentimiento, por el cual se recobra el favor de Dios. Se centra en la época medieval y renacentista, pero sin dar especial atención a ejemplos de la Península Ibérica. Huttar sostiene lo siguiente:

The name of David also occurs frequently in another list of examples, for the encouragement of all who, having fallen, are tempted to the still worse sin of despair. If such great offenders as David, Peter and Mary Magdalene could be restored through patience, there is surely hope for all (39).

Este estudioso argumenta que David se lee durante la Edad Media como un personaje bíblico que cuenta con el favor de Dios (entonces vence a Goliat), pero después se deja llevar por la lujuria y la soberbia, y sus pecados llegan incluso al asesinato (2 Samuel 12:9). Sin embargo, su arrepentimiento le vale de nuevo el favor divino y así recupera lo perdido. Las conclusiones del artículo de Huttar son significativas para nosotros, pues intentaremos, más adelante en este trabajo, relacionarlas con la suerte del pueblo castellano y de Fernán Gonzáles en el Poema:

This survey has shown the great pictorial and narrative variety that entered into the reshaping and embelishment of a biblical story -changing according to the emphases of piety and general culture in each age. But through all the changes remains the constant theme that the repentant and restored David, now spiritually atronger than ever, stands as an encouragement and guide to ordinary sin-prone men and women (54).

\section{La inferioridad numérica de los castellanos en las batallas narradas en el Poema}

En realidad, la historia del Poema se inicia antes de que el héroe haga su aparición. El propósito del narrador es llegar a Fernán Gonzáles, y esto está dicho desde la copla 1: “...del conde de Castiella quero fer una prosa...", pero para eso, su plan narrativo incluye la información anterior, según lo demuestra en la copla 5:

En tanto, desde tienpo ir vos he yo contando commo fueron la tierra perdiendo e cobrando,

fasta que todas fueron al conde don Fernando.

Vale la pena, pues, preguntarse si en las batallas narradas antes de que hiciera su aparición el conde, hay inferioridad numérica. Cuando se narra la pérdida de España, no hay motivos para pensar que los reyes godos estuvieran en tal situación, de acuerdo con el texto. La derrota de que son objeto se atribuye más bien a que, siendo muchos y dueños de la península, los godos deciden desarmarse. Léase la copla 77:

Quando ovo el rey Rodrigo sus poderes juntados era poder sin guisa, mas todos desarmados-, 
lidiar fueron con los moros, lavaron sus pecados,

ca fue de los profetas esto profetizado.

Aunque ha habido un consejo traidor del conde don Yllan, es finalmente el diablo quien asume la responsabilidad, y no se hace referencia alguna a las cantidades de enemigos que atacaron a los cristianos.

Lo que sí ocurre es que de la situación que esta derrota causa se desprende una disminución de la población cristiana. Léanse las coplas 94 y 95 :

Assi ivan foyendo de las gentes extrañas

murian de grand fanbre todos por las montañas, non diez, veinte nin treinta, mas muchas de conpañas.

Perdieron muchos d'ellos con miedo los sentidos, matavan a las madres, en braços a sus fijos, no s'podien dar consejo mugeres nin maridos, avian, con grand miedo, muchos enloquecidos.

Luego de la pérdida de España a manos de los moros, pero antes de la aparición del conde en el texto, tenemos la batalla de Roncesvalles, que vale la pena examinar. En primer lugar, las motivaciones de esta batalla son importantes pues implican un deseo de que Castilla permanezca como una nación sin influencia extranjera, así se trate de extranjeros cristianos, en este caso, franceses. Estos son muchos, de acuerdo con la copla 131: "Ayunto sus poderes, grandes e sin mesura, / movio pora Castiella, ..."

Sin embargo, no está claro, en esta batalla que ganan los castellanos, que estos se encuentren en situación de inferioridad numérica.

Antes de la aparición del conde se establece la pequeñez inicial de Castilla, en la copla 171 y principio de la 172 :

Entonçe era Castiella un pequeño rincon,

era de castellanos Montes d'Oca mojon,

e de la otra parte Fitero el fondon,

moros tenien Caraço en aquesta sazon.

Era toda Castiella solo una alcaldia...

Esa es la situación en que nace el conde Fernando, cuyo cometido expansionista en el texto se establece desde un principio. Resaltemos la acotación "con muy poca conpaña", importante para nuestra lectura, en las coplas 175 y 176 :

Fizo grandes batallas con la gent descreida,

e les fizo lazrar a la mayor medida,

ensancho en Castiella una muy grand medida,

ovo en el su tienpo mucha sangre vertida.

El conde don Fernando, con muy poca conpaña

-en contar lo que fizo semejarie fazaña-

montovo sienpre guerra con los reys d'España

non dava mas por ellos que por una castaña.

Pero pasemos ya a las batallas en las que el conde toma parte, la primera de las cuales es la toma de Carazo. No se habla en ella de las cantidades, por ambos bandos, de guerreros 
participantes. Pero esta derrota de los musulmanes genera un contrataque, en el cual Almanzor será vencido pese a su clara ventaja numérica. Veamos la copla 197:

Quando fueron con el juntados sus varones,

reyes e ricos omnes e muchos infançones,

si todos los contassemos, caveros e peones,

serian mas por cuenta de cinco mill legiones.

Así, los castellanos mismos no tienen más remedio que admitir su pequeñez frente a un enemigo gigantesco, y Fabio Gonzalo Díaz aconseja evitar el combate (coplas 205-206):

\footnotetext{
Muchos e sin guisa los pueblos renegados, caveros e peones todos bien aguisados; somos poca conpaña, de armas muy menguados, seremos, si nos vençen, todos descabeçados.

Si nos pleito podiessemos con Almançor tener, que fincasse la lid por dar o prometer, es el mejor consejo que podriemos aver; si otra cosa fazemos, podemos nos perder.
}

Pero el conde interviene y los castellanos pelean. Estudiaremos la retórica del conde más adelante. Por ahora, bástenos confirmar que los castellanos combaten en Lara en clara desventaja numérica, según la copla 251:

Otro dia mañana mando mover sus gentes; pora cada cristiano avie mill descreyentes; los del conde eran pocos, mas buenos conbatientes, todos eran iguales, d'un coraçon ardientes.

Estas condiciones numéricas y el resultado victorioso de los castellanos jefeados por Fernán Gonzáles se repiten en el primer enfrentamiento que tienen estos con los navarros. La superioridad cuantitativa está en las palabras del mismo Fernán Gonzáles, en las coplas 304 y 307 :
En nos lo cometer es nuestra mejoria, por quanto ellos son mayor cavalleria; nos non mostremos y ninguna covardia, en dudar nos por ellos serie grand villania. (...) Muchos son mas que nos peones e caveros, omnes son esforçados e de pies muy ligeros, d'asconas e de dardos fazen golpes çerteros traen buena conpaña de buenos escuderros.

Entran los castellanos en la llamada Batalla de la Era Degollada, y salen relativamente victoriosos. Agreguemos que cuando el conde de Tolosa desea vengar al rey navarro, muerto por mano de Fernán Gonzáles, de nuevo hay castellanos que prefieren evitar la batalla, y las razones son entre otras la situación desventajosa de ser menos, pues ni siquiera se encuentran presentes todos los guerreros castellanos. Así lo dice Nuño Laíno en la copla 354 y parte de la 346: 
Dexa folgar tus gentes, a ti mismo sanar, tienes muy fuerte llaga, dexa la tu folgar, dexa venir tus gentes, ca aun son por llegar, muchos son por venir, deves los esperar. Tu seras a diez dias del golpe bien guarido, sera ya el tu pueblo a esse plazo venido... castellanos.

De nuevo el conde exhortará al combate inmediato y de nuevo la victoria será de los

Más tarde, se narra la victoria de Hacinas. Almanzor ha vuelto a la carga con un gigantesco ejército, que reúne ahora a varias naciones musulmanas, según se lee en la copla 384 :

\footnotetext{
Que venie Almançor con muy fuertes fonsados, con çiento e treinta mill caveros lorigados, non serien los peones nulla guisa contados, estavan çerca Lara en Muño ayuntados.
}

Y es que aunque el conde logrará reunir un ejército grande, las cifras que se presentan en el bando cristiano son muy inferiores frente a las que acabamos de citar. Veamos las coplas 460, 461 y 463, 464:

\footnotetext{
Venien estos caveros en essa az mediana, estos eran dozientos de la flor castellana; todos fueron en campo otro dia mañana, essa fue pora moros una negra semana.

Dio les seis mill peones con que los conbatiessen,...

(...)

El conde don Fernando, de los fechos granados, ovo veinte escuderos en esse dia armados; estos con el buen conde en az fueron entrados, por todos son cinquenta, non mas fueron contados.

Venien tres mill peones, todos de buena gente,..
}

Hagamos cuentas, pues. Si los moros son ciento treinta mil, los cristianos, entre caballeros y peones, no serán siquiera diez mil, pese a lo cual vencerán, como David y Goliat.

Todavía quedan en el texto las narraciones de algunas batallas. Pero recordemos que a partir de la copla 570, el texto pasa a ocuparse de aspectos relativos más a lo político y lo privado en la vida del conde, que a lo militar, que hasta entonces dominaba. Las narraciones de las batallas que quedan no tienen la fuerza que han tenido las anteriores: se les dedica mucho menos espacio y se explicitan menos detalles de las condiciones de lucha. No encontramos en ellas rastros de datos numéricos; si bien en ningún caso se establece la desventaja numérica, tampoco se dice lo contrario.

\section{Las arengas del conde y las intervenciones divinas, claves de las victorias castellanas}

Al darse el enfrentamiento con Almanzor, en la batalla de Lara, los castellanos se presentan cautos, como ya hemos visto, y es la arenga de Fernán Gonzáles la responsable de que 
entren en combate. Esta arenga se encuentra en las coplas 209 a 224, de manera que tiene una extensión considerable. Estudiemos los puntos de que consta esta intervención.

En primer lugar, hay una referencia a la muerte, que se presenta como el destino inevitable de todos los seres humanos. Pero, al mismo tiempo, el conde recuerda la muerte del Salvador, Jesucristo, quien muere por combatir el engaño, y genera así el compromiso religioso de sus oyentes, en el sentido de no dudar en ofrendar la vida, como lo hizo el Dios Hijo, con tal de defender la verdad, que en este caso se homologa con la causa castellana. Vemos así el artificio retórico: nunca se discute si la causa castellana realmente coincide con los ideales de Jesucristo, esto se da por hecho, como "verdad natural".

En segundo lugar, el conde recurre al recuerdo de la historia de Castilla, pues así genera un doble compromiso en sus oyentes. Estos deberán combatir no sólo por las razones religiosas antes expuestas, sino porque los antepasados así lo hicieron. Y el conde no esconde que la lucha antigua se dio también en situación de inferioridad numérica, como se ve en la copla 218:

\footnotetext{
Fueron nuestros abuelos muy grand tiempo afrontados ca los tenien los moros muy fuerte arrenconados; eran en poca tierra pocos omnes juntados, de fanbre e de guerra eran mucho lazrados.
}

El conde apela al estoicismo y al espíritu de sacrificio de los oyentes presentes al recordarles una idéntica conducta en los antepasados. He aquí el artificio retórico: tal conducta de los antepasados es una elaboración imaginaria del conde, quien no aporta prueba alguna al respecto.

Finalmente, el argumento capital del conde es la confianza en la victoria, y para esto su punto es la superioridad de los castellanos en relación con sus enemigos. A la superioridad cuantitativa, opone el conde la superioridad cualitativa. Así se ve en la copla 223 (el tercer verso es reconstrucción del editor):

Maguer que muchos son, non valen tres arvejas

mas pueden tres leones que treinta mil golpejas irian treinta lobos a treinta mil ovejas.

De la victoria, dada por segura, extrae el conde la consecuencia del acrecentamiento de la honra propia y de los castellanos. He aquí el artificio retórico: el conde muestra la recompensa como un hecho, pasando por alto el que este es el efecto de una causa que aún no se ha dado: la batalla todavía no se da, y la victoria es dudosa. Se podría aplicar aquí el refrán que dice que el conde venda la piel del león antes de cazarlo. Y sus hombres se la compran...

Así pues, la organización de la arenga del conde tiene tres partes, la última de las cuales es la más positiva, pues resalta los logros de la victoria, y es esto lo que el conde quiere dejar en los oídos de sus oyentes, más que el sacrificio de Cristo o el que los moros son muchos más que los castellanos. No hay duda de que Fernán Gonzáles sabe en esta ocasión cómo arengar a la tropa.

Hay una nueva arenga del conde antes de la batalla de Lara, según la reconstrucción que se ha hecho del texto, pues las coplas 257 a 260 están perdidas. Esto, claro, nos impide llevar a cabo un análisis de esta intervención de Fernán Gonzáles. 
En la batalla primera contra los navarros también se presenta una arenga del conde. Tenemos en total diez coplas, de la 300 a la 310. Aquí la intervención del conde comienza por plantear la oposición entre la conducta recta de los castellanos frente a los navarros y la agresión que estos han llevado en contra de los primeros. Así dice la copla 301:

\footnotetext{
Nunca a los navarros mal non les feziemos, nin tuerto nin sobervia nos nunca les feziemos; muchos fueron los tuertos que d'ellos resçibiemos, por gelo demandar nunca sazon toviemos.
}

Como hemos visto en las coplas 304 y 307, ya transcritas, el siguiente argumento del conde consiste en admitir la superioridad numérica del enemigo, pero al mismo tiempo la relativiza, pues pide a sus hombres que no muestren miedo en la batalla e incluso que tomen la iniciativa, lo cual servirá para debilitar al enemigo numeroso. Veamos la copla 308:

\footnotetext{
Por esto ha mester que nos los cometamos;

si ellos nos cometen, mejoria les damos;

si ellos entendieren que nos non los dubdamos,

dexar nos han el campo antes que los firamos.
}

No hay duda de que el razonamiento, aunque podría ser útil como artificio retórico pues este es el arte de convencer, no de razonar derechamente- es débil: lanzarse contra el otro pese a la superioridad numérica de este pues así huirá. Pero, ¿qué nos asegura que el otro tendrá la conducta prevista, cuando somos nosotros los menos?

Por esto quizá no es esta la más acabada lección de retórica, ya que incluso el conde entrevé, al final de la arenga, la posibilidad de su muerte, y esta tampoco es la mejor forma de motivar a sus hombres. Esta batalla, acaso en parte por la debilidad de la arenga, es de un éxito relativo, pues el conde es herido.

Al enfrentarse poco después al conde de Tolosa, el conde pasa de nuevo a arengar a los suyos. Tiene que desautorizar a Nuño Laínez, como antes ha desautorizado a Gonzalo Días. Pero no escoge el enfrentamiento total, como hizo antes, sino que esta vez da en parte la razón a su oponente retórico. Su punto es, sin embargo, que no hay que perder un solo momento, ni siquiera por prudencia, pues la honra solo se gana con acciones. Aquí es cuando se compara con David, según hemos acotado ya (copla 354). El artificio retórico es similar a uno utilizado antes: mostrar la recompensa por la acción cuando esta todavía no se ha realizado.

Al darse el nuevo enfrentamiento con Almanzor, en Hacinas, aparece también una arenga del conde. Es sumamente extensa: va de la copla 424 a la 447 . Se inicia de una manera particular: el conde narra a sus hombres cómo se le ha manifestado a él personalmente la voluntad de Dios a través del monje San Pelayo, en una especie de visión. Esto es notable: la intervención divina tiene que complementarse con la narración de esa intervención, dentro del mundo narrativo. Veamos las coplas 427, 428 y 429:

\footnotetext{
...vino a mí este monje en una vision:

"Despierta -dixo- amigo, que ora es e sazon."

Dixo me lo en sueños, non lo quise creer,

desperte e non pude ninguna cosa ver;

oi una grand voz del çielo descender.
} 


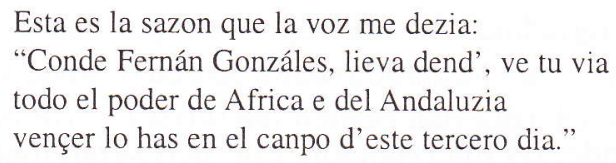

Asegurada la victoria por esta información sobrenatural, nada parece mejor al conde que amenazar con el castigo -no terrenal, sino divino- a quienes se atrevan a desobedecerle, pues no se trata ya de desobedecer al conde, sino a Dios mismo. La segunda parte de la arenga establece la superación del temor a la muerte, siempre y cuando no se muera como traidor (aquí se evoca la figura de Judas), lo que equivaldría a ganarse el infierno. Tenemos como prueba la copla 447:

\footnotetext{
Todo aquel que de vos del canpo se saliere con miedo de muerte a presion se les diere, quede por alevoso si tal fecho fiziere,

con Judas en infierno yaga cuando moriere.
}

Toda esta intervención se resume en un manejo imaginario de la realidad, pues se la presenta como ordenada por fuerzas sobrenaturales, cuyo conocimiento sólo tiene el conde: los otros han de aceptar lo que Fernán Gonzáles tenga que decir al respecto. Agreguemos tan sólo que dentro de la arenga del conde se incluye una aceptación de la inferioridad numérica de su ejército, en la copla 435 ("Mill y ha pora uno, esto bien lo sabemos,...").

Hay una nueva arenga, el segundo día de combate, pero se ha perdido (coplas 522 a 525). La reconstrucción permite saber que su contenido era también religioso, pero es imposible su análisis.

Pasemos ahora al estudio de las intervenciones divinas, para abarcar con ello el segundo factor que produce la victoria castellana. Recordemos, en primer lugar, que España se ha perdido por culpa del diablo. Leamos la copla 70:

\footnotetext{
Ovieron a fer todo lo que el rey les mandava, quien las armas tenia luego las desatava: el diablo antiguo en esto s'trabajava, por fer mal a cristianos, nunca en al andava.
}

Pero esto no quiere decir que Dios no haya intervenido, como lo demuestra la copla 80:

Era la cosa puesta e de Dios otorgada que serien los de España metidos a espada: a los dueños primeros les serie tomada. Tornaron en el canpo ellos otra vegada.

Y sabemos, aunque el Poema no pone ahí el énfasis, que Rodrigo ha cometido un pecado de lujuria -su relación con la Cava- y por ello Dios lo está castigando. Veamos lo que dice la copla 35:

Fino se Vautiçanos, reino rey don Rodrigo: avien en el los moros un mortal enemigo; 
era de los cristianos sonbra e grand abrigo;

por culpa en que era, non le era Dios amigo.

En las batallas en que participa el conde, las intervenciones divinas serán mucho más importantes. No las hay, sin embargo, en la toma de Carazo. Pero luego estará muy claro de parte de quiénes se encuentra el poder celestial. Entre las coplas 226 y 248 , se narra el episodio en que el conde se abstiene de profanar un lugar sagrado, el encuentro con Pelayo y la promesa que este hace -como mensajero de Dios- en relación con el triunfo de los castellanos. Luego de la victoria inicial, el conde hace su donación a San Pedro de Arlanza, con lo que se termina de concretar, en acciones específicas (valga la redundancia), el ciclo en que se suceden la piedad del conde, la promesa de ayuda divina, el triunfo militar y el pago por la ayuda que se hace a Dios por medio de la iglesia.

En la narración de la victoria de Hacinas, tenemos primero la oración del conde (coplas 394 a 404), que inmediatamente es respondida por Dios, quien se manifiesta en sueños por medio del monje San Pelayo y le promete la ayuda solicitada (coplas 405 a 412). Pero no basta un enviado: entre las coplas 414 y 418, don Millán reitera las instrucciones militares de Jesucristo. Veamos la copla 418:

\section{Non quiero mas dezir: lieva dend', ve tu via. ¿Quieres saber quien trae esta mensajeria? Millan so yo por nonbre, Jesucristo me enbia, durara la batalla fasta terçero dia.}

Como hemos constatado, el conde no se abstiene de incluir estos episodios en sus arengas, para infundir valor a sus hombres. Y en realidad, cuando el diablo aparece en la batalla (coplas 467 a 470), Fernán Gonzáles sabe inmediatamente de quién se trata y no duda de que vendrá la ayuda de Dios, según se puede ver en la copla 480:

Como sodes sesudos, bien podedes saber que non ha el poder de mal a nos fazer, ca tollo le don Cristus el su fuerte poder, veades que son locos los que l'quieren creer.

Poco después, ocurre un episodio que se parece mucho a la narración de las Sagradas Escrituras, pues aparece un jefe moro gigantesco, cual Goliat. Léase la copla 491:

Un rey de los de Africa era y cavalgante, -entre todos los otros semejava giganteque al conde buscava, -e el conde al semejante-; luego quando lo vio fue se l'parar delante.

El conde se enfrenta a este "Goliat"y lo mata; luego pide la ayuda de Dios para repeler a los moros que tratan de vengar al gigante caído. Es curioso cómo, aunque no hay ninguna intervención directa de Dios (pues el conde se salva gracias al caballo que le proporcionan sus vasallos en el último minuto), el conde da gracias a Dios, con lo que lee la realidad de una manera imaginaria. 
Nada hay de imaginario, sin embargo, cuando viéndose en mala situación vuelve el conde a pedir ayuda de Dios (coplas 547 a 555) y aparece al apóstol Santiago. (Hablamos, claro, en el nivel interno del mundo narrado). El texto ofrece aquí una prueba irrebatible, con carácter de milagro, de que Dios apoya a los suyos, los castellanos. Es el momento culminante de las intervenciones divinas.

Queremos destacar, en este estudio de las arengas y las intervenciones divinas, que estas últimas siempre se incorporan, como material narrado, a las primeras. El conde no guarda en secreto lo que le ha ocurrido; podríamos sospechar que el personaje funciona a sabiendas de que un milagro no es tal sin una metanarración que lo diga. El texto descubre así, a nuestros ojos, su propia forma de funcionamiento: el Poema dice sus hechos también, como el conde, para que las verdades se consoliden a través de la palabra.

\section{Conclusiones}

Realizado el análisis anterior, nos parecen claros los siguientes hechos:

1. Los castellan'os, por regla general, combaten en situación de inferioridad numérica en el Poema, y aun así vencen.

2. El conde, en el nivel personal, también tiene el poder de imponerse a enemigos más numerosos o más corpulentos.

3. El texto establece la ayuda de la Divinidad a los castellanos, y la oposición demoniaca a estos, durante las batallas, lo que se traduce directamente en victorias. Solo por ausencia de esta ayuda, en la época de Rodrigo, fue este grupo derrotado.

4. Las arengas del conde son imprescindibles, dentro del mundo narrado, como preparación para las batallas en que los castellanos participan y triunfan.

5. La comparación entre el personaje bíblico David y el conde de Castilla es válida, tanto desde el punto de vista del contexto cultural como por las evidencias textuales y los estudios anteriores.

Así pues, el personaje del conde Fernán Gonzáles se escribe teniendo como modelo entre otros que no se han contemplado en este estudio- la figura bíblica de David, quien con la ayuda de Dios vence a Goliat. La elección de este modelo se explica si tenemos en cuenta el proyecto expansionista castellano, presente en el cantar perdido, y retomado en el Poema durante el siglo XIII, cuando nuevos proyectos expansionistas -o, si se quiere, la continuación de los viejos- están en agenda. David representa, dentro de la Biblia, el poder expansionista del pueblo hebreo, que crece venciendo a enemigos más poderosos, pues cuenta con el apoyo de Dios (1 Samuel 17: 4-55).

Pero podemos ir más allá. Se ha señalado que el poder de Dios que se da a David para vencer a Goliat y convertirse en líder, se ve puesto en peligro cuando David peca, y no se asegura de nuevo sino cuando este se arrepiente. Podemos relacionar esto con la historia de Fernán Gonzáles y los cristianos, pero haciendo la salvedad de que el pecado no lo comete este personaje, sino Rodrigo. Los españoles pierden la Península por el pecado de este, pero luego la retomarán, con la ayuda de Dios, cuando con Fernán Gonzáles son capaces de apartarse 
del pecado y luchar de nuevo. (Recuérdese que es el diablo el que propende al pacifismo, según se ve en el episodio del desarme de los castellanos.) Fernán Gonzáles, como Cristo, salvará al pueblo del yugo del enemigo, que se homologa a Satán.

Todo esto produce un efecto fuera del mundo narrado, es decir, en el nivel de los lectores, que es quizá lo principal. El receptor del siglo XIII entiende, por medio de la homologación entre el personaje bíblico David y el personaje histórico-literario Fernán Gonzáles, su propio compromiso religioso de la Reconquista, su propia necesidad de enfrentarse sin dilación alguna al enemigo, así sea este más poderoso, y con toda confianza, pues el apoyo de Dios es indudable. Del plano de la fe se deriva una consecuencia político-militar que hace palpable el propósito primordial del texto.

\section{Bibliografía}

1967. La Biblia. Traducción de Watchtower and Tract Society of New York, Inc. Nueva York: Internacional Bible Students Association.

Cotrait, René. 1973. "Pour une bibliographie de Fernán Gonzáles. III: Un passage oublié du David livre de Samuel, source possible d'un épisode de la légende de Fernán Gonzáles (variété). Bulletin Hispanique. LXXV (3-4): 359-82.

Frontain, Raymond-Jean and Jan Wojcik. 1980. "Introduction: Transformations of the Myth of David". The David Myth in Western Literature. West Lafayette, Indiana: Purdue University Press: 1-11.

Huttar, Charles A. 1980. "Frail Grass and Firme Tree: David as a Model of Repentance in the Middle Ages and Early Renaissance". The David Myth in Western Literature. West Lafayette Indiana: Purdue University Press: 38-54.

Lacarra, María Eugenia. 1979. "El significado histórico del Poema de Fernán Gonzáles”. Studi Ispanici III: 9-41.

O’Callagham. 1992. "Introducción” Prosa hsitórica de Alfonso el Sabio, Madrid: Cátedra.

Riché, Pierre. 1984. "La Bible et la vie politique dans le haut Moyen Age”. Le Moyen Age et la Bible. Paris: Beauschesne: 385-400.

Victorio, Juan (ed.). 1998. Poema de Fernán Gonzáles. Madrid: Cátedra.

1998. "Introducción”. Poema de Fernán Gonzáles. Madrid: Cátedra.

Smalley, Beryl. 1952. The Study of the Bible in the Middle Ages. London: Basil Blackwell Publisher Limited. 